\title{
Authors' Reply to Montoya et al. Comment On: “Tools Measuring Quality of Death, Dying, and Care, Completed After Death: Systematic Review of Psychometric Properties"
}

\author{
Nuriye Kupeli ${ }^{1}$ (1) - Bridget Candy ${ }^{1}$ - Gabrielle Tamura-Rose ${ }^{2}$. Guy Schofield ${ }^{3} \cdot$ Natalie Webber $^{4} \cdot$ Stephanie E. Hicks $^{5}$. \\ Theodore Floyd $^{6} \cdot$ Bella Vivat $^{1} \cdot$ Elizabeth L. Sampson $^{1,7} \cdot$ Patrick Stone $^{1} \cdot$ Trefor Aspden $^{8}$
}

Published online: 3 December 2018

(c) Springer Nature Switzerland AG 2018

\section{Dear Editor-in-Chief,}

We thank Montoya and colleagues [1] for taking the time to read our paper "Tools measuring quality of death, dying, and care, completed after death: Systematic review of psychometric properties" [2] and drawing our attention to their related study. We acknowledge that the short version of the Good Death Inventory may have some positive psychometric properties. Our review identified one paper by Miyashita and colleagues [3] entitled "Good death inventory: a measure for evaluating good death from the bereaved family member's perspective", which explored the psychometric properties of the short version of the Good Death Inventory in the population of interest. This paper reported good internal consistency (Cronbach's alpha 0.85) and reliability (intraclass

This reply refers to the comment available at: https://doi. org/10.1007/s40271-018-0350-4.

Nuriye Kupeli

n.kupeli@ucl.ac.uk

1 Marie Curie Palliative Care Research Department, Division of Psychiatry, University College London, London, UK

2 Department of Palliative Care, Royal Free Hospital, London, UK

3 Centre for Ethics in Medicine, University of Bristol, Bristol, UK

4 Royal Trinity Hospice, London, UK

5 St Christopher's Hospice, London, UK

6 Emergency Department, Medway Maritime Hospital NHS Trust, Kent, UK

7 Barnet Enfield and Haringey Mental Health Trust Liaison Psychiatry Team, North Middlesex University Hospital, London, UK

8 Division of Psychiatry, University College London, London, UK correlation 0.71 ); however, this study used data collected to assess the psychometric properties of the long version of the Good Death Inventory to develop and assess the properties of the short version. We hope this clarifies our findings and recommendations for more research evaluating the psychometric properties of these tools.

\section{Compliance with Ethical Standards}

Funding The improving care, assessment, communication and training at the end-of-life (I-CAN-CARE) programme is funded by Marie Curie Cancer Care (Grant reference: MCCC-FPO-16-U).

Conflict of interest Nuriye Kupeli, Bridget Candy, Gabrielle TamuraRose, Guy Schofield, Natalie Webber, Stephanie E. Hicks, Theodore Floyd, Bella Vivat, Elizabeth L. Sampson, Patrick Stone and Trefor Aspden have no conflicts of interest that are directly relevant to the contents of this letter.

\section{References}

1. Montoya-Medina JE, Jabbour GP, Urrunaga N, Jiménez HA. Comment on: Tools Measuring Quality of Death, Dying, and Care, Completed After Death: Systematic Review of Psychometric Properties. Patient. 2018. https://doi.org/10.1007/s4027 1-018-0350-4.

2. Kupeli N, Candy B, Tamura-Rose G, et al. Tools measuring quality of death, dying, and care, completed after death: systematic review of psychometric properties. Patient. 2018. https://doi. org/10.1007/s40271-018-0328-2 (epub ahead of print).

3. Miyashita M, Morita T, Sato K, et al. Good death inventory: a measure for evaluating good death from the bereaved family member's perspective. J Pain Symptom Manag. 2008;35(5):486-98. 